\title{
Creation through Polychronization
}

\author{
John Matthias \\ School of Art, Design and Architecture, \\ Plymouth University, UK \\ john.matthias@plymouth.ac.uk
}

Received 2 June 2017; accepted 30 September 2017; published 21 November 2017.

\begin{abstract}
I have recently suggested that some of the processes involved in the collaborative composition of new music could be analogous to several ideas introduced by Izhikevich in his theory of cortical spiking neurons and simple memory, a process which he calls Polychronization. In the Izhikevich model, the evocation of simple memories is achieved by the sequential re-firing of the same Polychronous group of neurons which was initially created in the cerebral cortex by the sensual stimulus. Each firing event within the group is contingent upon the previous firing event and, in particular, contingent upon the timing of the firings, due to a phenomenon known as "Spike Timing Dependent Plasticity." I argue in this article that the collaborative creation of new music involves contingencies which form a Polychronous group across space and time which helps to create a temporary shared memorial space between the collaborators.
\end{abstract}

Keywords: collaboration; composition; polychronization; spike timing dependent plasticity.

\section{Hodgkin and Huxley}

In a remarkable paper written in 1976, the biologist Alan Hodgkin describes some of the accidents and designs which led to the discovery of the mathematical physics of the electric current transport through the membrane of the axonal nerve fiber of the longfin inshore squid (Hodgkin, 1976), a discovery which culminated in four papers in 1952 written with Andrew Huxley (Hodgkin \& Huxley, 1952) for which they were awarded the Nobel Prize in Physiology or Medicine in 1963 and which still underpin our knowledge of the mathematical physics of the dynamics of neurons. Hodgkin's 1976 paper begins with him as an undergraduate student in Cambridge in 
the 1920s working with self-built amplifiers in a second-year laboratory, takes us through collaborative conversations and experiments with colleagues in Cambridge, New York, St. Louis and Plymouth, down blind alleys and through joyous discoveries and disappointments including the final admission of finding their initial hypothesis difficult to prove which meant that, in Hodgkin's words, "we settled for the more pedestrian aim of finding a simple set of mathematical equations which might plausibly represent the movement of electrically charged particles" (Hodgkin, 1976, p. 19). This "simple set of mathematical equations" are now the mathematical cornerstone of brain physics and form the basis for all dynamical discussion of the interaction between neurons.

In the early 21 st century, much of the mathematical biological community interested in brain research was focused on the behavior of large networks of neurons and due to the many variables and highly non-linear nature of the Hodgkin-Huxley ( $\mathrm{HH})$ equations, simpler equations had to be used to model the very large networks as computing power was not sufficient to calculate the large network dynamics. There are several simplifications of the $\mathrm{HH}$ equations, the most simple of which reduce the four variables of $\mathrm{HH}$ to a single membrane voltage (the integrate-and-fire model; Lapicque, 1907) and others which reduce the four variables to two interconnected variables (e.g., the Moris-Lecar model; Morris \& Lecar, 1981) and the FitzHughNagumo model (FitzHugh, 1955; Nagumo, Arimoto, \& Yoshizawa, 1962). One such model is the Izhikevich model (Izhikevich, 2003) which computes the firing times and firing patterns of many connected neurons and which also incorporates the more recently discovered effect of spike timing dependent plasticity (STDP; Song, Miller, \& Abbot, 2000), an effect which shows that the synaptic strength of connection between neurons depends upon the relative timing of the arrival of neural signals at the synaptic junctions. One of the main outcomes of the Izhikevich model is the concept of "Polychronization," a term coined by him (Izhikevich, 2006) to describe the dynamics underlying the formation of neural pathways through the mammalian cerebral cortex. The idea is that STDP and the neural firing dynamics conspire to produce a unique polychronous group of firing neurons for each sensory stimulus, and that we can evoke (re-imagine) the original stimulus by reproducing the firing of this polychromous group. The mechanism for the re-imagining remains one of the mysteries of conscious behavior.

\section{Collaboration}

In an informal recollection of some of the processes used in the collaboration between myself and Jay Auborn in the making of the music album Race to Zero (Matthias \& Auborn, 2017), I have suggested (Matthias, 2015) that Izhikevich's idea of Polychronization (extended over weeks, months and years, and between people, not cells) provides an interesting analogy for collaborative artistic creation. I suggest 
that the sound of one of our tracks, "Songbird," depends on the contingencies of many events, from the agreement by 17 th-century luthiers that the violin strings should be a fifth apart, to the jerky movements of the Company Chameleon dancers (which informed the bowing technique used to create the earlier track, "Birdsong" 1 ), to the impressions that Jay and I were doing of Miles Davis in a conversation in Jay's kitchen about the Dorian mode. Each of these events is a kind of "firing," and the track "Songbird" could be said to have its own polychronous group of firing events which led to its unique creation. One attractive quality of this idea is that it implicitly includes the notion of context in artistic creation. There are many contexts in the creation of each collaborative "firing" event, including the economic cultural context of the record company deciding to fund the recording of the album. The creation through Polychronization idea is, in a sense, an extension of David Byrne's arguments regarding the co-evolution of music and architecture (Byrne, 2012, 2017) in which he suggests that musical and acoustic sensibilities co-evolved such that Bach's music, for example, would not have been possible if the acoustic of the church in Leipzig where Bach's music was performed had not allowed for modulations in key during a piece. Byrne's ideas relate to the interdependence of two factors, music and architecture. Here, I am suggesting that the polychronous creation model takes into account the importance of the co-evolution of music styles with architecture but also includes the many other contingent events and contexts which accompany a musical creation, such as economic and technological contexts and whether one person met another at a party in 1956. Furthermore, because the idea of spike timing dependent plasticity is inherently implicit in the formation of Polychronous groups, the theory I am proposing is inherently dynamic and includes a kind of social "Spike Timing Dependent Plasticity": Ideas that happen at the right time tend to catch on (strengthen the social synapses) if the contexts and the ideas line up; if they do not and the time is not right, they tend not to attach. This leads naturally to Polychronous contingency. In Spike Timing Dependent Plasticity, the relative timing of signals dictates whether the synapse between pre-synaptic and post-synaptic neuron will be enhanced or weakened; indeed, the change in synaptic strength depends exponentially upon the relative arrival time and drops off within milliseconds. It would be very interesting to examine whether there is a similar functional form within the model I am proposing here (with a time-scale is likely to be months or years rather than milliseconds).

\section{Betweenness}

There is another sense in which Izhikevich's concept of Polychronization can be applied to collaborative creativity which is linked with the network of ideas and experiences which evolve between two (or more) creators. Conversations and actions between collaborators form a kind of inbetween world which becomes a reference

\footnotetext{
${ }^{1}$ Listen to fragments of "Songbird" and "Birdsong" at: https://doi.org/10.26913/80s02017.0111.0015
} 
point for all further activity. This means that the network of Polychronous cellular activity goes beyond the brains of each collaborator and resides in both and between both. The experience of collaboratively improvising a certain piece or having a conversation about a particular piece of music creates this between-world which is a combination of contexts and occurrences. Reading the account by Hodgkin (1976) also makes it clear that the ideas which I am suggesting also take place within scientific enquiry as well as musical creation. Hodgkin writes about using a particular piece of experimental kit, partly because of his acquaintance with the inventor (Lucas) as a child and because of his relationship with Hodgkin's father and is clear about the contingencies of certain lines of thinking upon particular conversations as well as on the (possible) results of scientific experiments.

"I believe that the record of published papers conveys an impression of directness and planning which does not at all coincide with the actual sequence of events ... Inwriting papers, authors are encouraged to be logical, and, even if they wished to admit that some experiment which turned out in a logical way was done for a perfectly dotty reason, they would not be encouraged to 'clutter up' the literature with irrelevant personal reminiscences" (Hodgkin, 1976, p. 1).

As Sue Denham has suggested, whether an idea catches on and becomes part of an artistic creation might depend upon this "inbetweenness": "A social mechanism of similar functionality might map well onto the example of Hodgkin and Huxley and the fortuitousness of invention. STDP picks out temporal coincidences without prior preconceptions and therefore can discover unpredicted connections simply on the basis of co-occurrence ... in some sense the social STDP should work like that too ... operating on anything and everything" (S. L. Denham, personal communication).

\section{References}

Byrne, D. (2012). How music works. San Francisco, CA: McSweeney's.

Byrne, D. (2017, February). How architecture helped music evolve [Video file]. Retrieved from https://www.ted.com/talks/david_byrne_how_architecture_helped_music_evolve

FitzHugh, R. (1955). Mathematical models of threshold phenomena in the nerve membrane. Bulletin of Mathematical Biophysics, 17(4), 257-278. doi:10.1007/BF02477753

Hodgkin, A. L. (1976). Chance and design in electrophysiology: An informal account of certain experiments on nerve carried out between 1934 and 1952. The Journal of Physiology, 263(1), 1-21. doi:10.1113/jphysiol.1976.sp011620

Hodgkin, A. L., \& Huxley, A. F. (1952). A quantitative description of membrane current and its application to conduction and excitation in nerve. The Journal of Physiology, 117(4), 500-544.

Izhikevich, E. M. (2003). Simple model of spiking neurons. IEEE Transactions on Neural Networks, 14(6), 1569-1572. doi:10.1109/TNN.2003.820440 
Izhikevich, E. M. (2006). Polychronization: Computation with spikes. Neural Computation 18(2), 245-282. doi:10.1162/089976606775093882

Lapicque, M. L. (1907). Recherches quantitatives sur l'excitation électrique des nerfs traitée comme une polarization [Quantitative studies on electric of nerves treated as polarization]. Journal de Physiologie et Pathologie Général, 9, 620-635.

Matthias, J. R. (2015). From Birdsong to Songbird: An adventure in collaborative creativity. Technoetic Arts, 13(3), 309-315. doi:10.1386/tear.13.3.309_1

Matthias, J. R. (Composer), \& Auborn J. L. (Composer, producer). (2017). Race to zero [CD]. London, UK: Village Green.

Morris, C., \& Lecar, H. (1981). Voltage oscillations in the giant barnacle muscle fiber. Biophysics Journal, 35(1), 193-213. doi:10.1016/S0006-3495(81)84782-0

Nagumo J., Arimoto S., \& Yoshizawa, S. (1962). An active pulse transmission line simulating nerve axon. Proceedings of the IRE, 50(1), 2061-2070. doi:10.1109/JRPROC.1962.288235

Song, S., Miller, K. D., \& Abbot, L. F. (2000). Competitive Hebbain learning through spike-timing-dependent plasticity. Nature Neuroscience, 3(9), 919-926. doi:10.1038/78829

\section{Appendix}

This paper was discussed by Liam Maloney (University of York) and Thomas Wennekers (Plymouth University) who had been asked to give a response. Liam Maloney raised the issue of Lapalace's demon in relation to the above ideas, adding that he was concerned about issues of agency within the argument being put forward. I responded by suggesting that even though one might be able to look at situations and contexts which have occurred over many time and space scales to form a creative idea or object looking back, I was not suggesting that all creative ideas were inevitable given a certain set of circumstances, but rather that one should consider context and circumstance when understanding what has happened and what might happen. Thomas Wennekers responded to the idea of Polychronization scientifically, going through the argument given by Izhikevich systematically, considering the argument presented above and questioning whether there might be a correspondence between the two. Thomas was open to the ideas and considered that many of the ideas in the above argument mapped to the Izhikevich ideas, although he was skeptical about the mapping of the mathematical of STDP but felt that the ideas which I have been outlining might be more akin to "Long Term Potentiation." There was an interesting discussion about whether the catching on of ideas over time might indeed follow an exponential dependence, as is the case with STPD. Indeed, as the exponential function is defined as the solution to a differential equation in which the rate of change of a quantity is proportional to the quantity itself, it might well find a correspondence. This will be explored in future work. 